\title{
An approach to non-radiodense aspirated foreign bodies in the paediatric patient
}

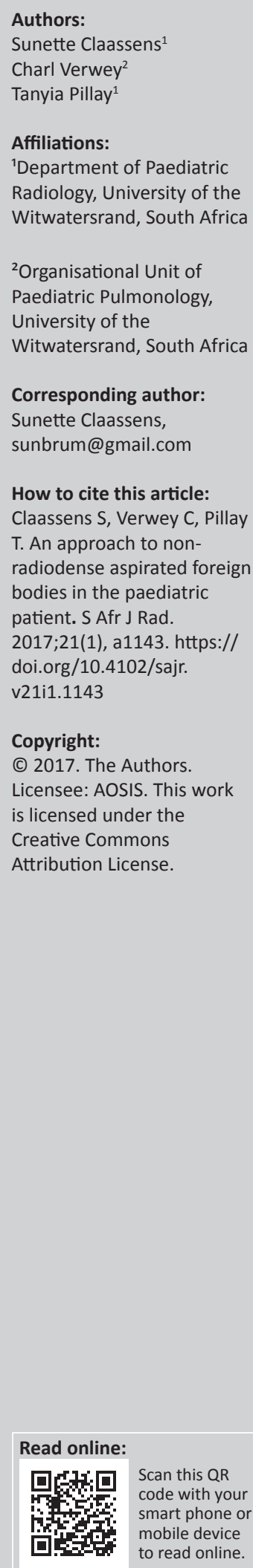

\section{Copyright:}

(c) 2017. The Authors.

Licensee: AOSIS. This work

is licensed under the

Creative Commons

Attribution License.

Introduction: Foreign body aspiration is a common and often serious problem in paediatric patients. Approximately $80 \%$ of paediatric foreign body aspiration episodes occur in children younger than 3 years. Almost $90 \%$ of aspirated foreign bodies are non-radiodense and therefore not seen on radiographs. Clinical presentation may be subtle and can become a diagnostic dilemma, particularly if there is no witnessed aspiration.

Aim: An educational poster on a radiological approach to aspirated non-radiodense foreign bodies.

Methods: We present serial chest radiographs of bronchoscopically confirmed non-radiodense foreign bodies.

Conclusion: Knowledge about the different radiological features suspicious for foreign body aspiration is essential and can guide appropriate intervention including bronchoscopic retrieval, which may prevent permanent pulmonary sequelae.

Note: A selection of conference abstracts: RSSA/SASPI Paediatric Imaging Congress, 03-06 November 2016, Spier Estate, Stellenbosch, South Africa. Faculty collaborators: Professor Kassa Darge (Body Imaging, University of Pennsylvania, Philadelphia,USA), Professor Edward Lee (Thoracic Imaging. Harvard University, USA), Professor Beverley Newman (Cardiac Imaging, Stanford University, California, USA), Professor Kimberly Applegate (Image Gently and Body Imaging, Emory University, Atlanta, USA) and Professor Savvas Andronikou (Thoracic Imaging, University of Bristol, UK) supported by South African Paediatric Radiologists, co-ordinated by Dr Jaishree Naidoo, President of the African Society of Paediatric Imaging and Head of Division of Paediatric Radiology, Charlotte Maxeke Johannesburg Academic Hospital. 